A complete electronic version of this article and other services, including high-resolution figures, can be found at:

http://stm.sciencemag.org/content/early/2012/11/12/scitransImed.3004190

Information about obtaining reprints of this article or about obtaining permission to reproduce this article in whole or in part can be found at:

http://www.sciencemag.org/about/permissions.dtl 


\title{
Optogenetic and Potassium Channel Gene Therapy in a Rodent Model of Focal Neocortical Epilepsy
}

\author{
Robert C. Wykes, ${ }^{1 *}$ Joost H. Heeroma, ${ }^{1 *}$ Laura Mantoan, ${ }^{1 *}$ Kaiyu Zheng, ${ }^{1}$ \\ Douglas C. MacDonald, ${ }^{2}$ Karl Deisseroth, ${ }^{3}$ Kevan S. Hashemi, ${ }^{4}$ Matthew C. Walker, ${ }^{1+}$ \\ Stephanie Schorge, ${ }^{1+}$ Dimitri M. Kullmann ${ }^{1+}$
}

\begin{abstract}
Neocortical epilepsy is frequently drug-resistant. Surgery to remove the epileptogenic zone is only feasible in a minority of cases, leaving many patients without an effective treatment. We report the potential efficacy of gene therapy in focal neocortical epilepsy using a rodent model in which epilepsy is induced by tetanus toxin injection in the motor cortex. By applying several complementary methods that use continuous wireless electroencephalographic monitoring to quantify epileptic activity, we observed increases in high frequency activity and in the occurrence of epileptiform events. Pyramidal neurons in the epileptic focus showed enhanced intrinsic excitability consistent with seizure generation. Optogenetic inhibition of a subset of principal neurons transduced with halorhodopsin targeted to the epileptic focus by lentiviral delivery was sufficient to attenuate electroencephalographic seizures. Local lentiviral overexpression of the potassium channel Kv1.1 reduced the intrinsic excitability of transduced pyramidal neurons. Coinjection of this Kv1.1 lentivirus with tetanus toxin fully prevented the occurrence of electroencephalographic seizures. Finally, administration of the Kv1.1 lentivirus to an established epileptic focus progressively suppressed epileptic activity over several weeks without detectable behavioral side effects. Thus, gene therapy in a rodent model can be used to suppress seizures acutely, prevent their occurrence after an epileptogenic stimulus, and successfully treat established focal epilepsy.
\end{abstract}

\section{INTRODUCTION}

Epilepsy affects more than 50 million people (1). Even with optimal drug treatment, more than $20 \%$ of people with epilepsy continue to have seizures (2). Intractability is especially common in localizationrelated (focal onset) epilepsy (3), where seizures arise from a region or a neuronal network that is limited to one hemisphere. Such regions are characterized by abnormalities of anatomy, neuronal connectivity, synaptic transmission, and/or intrinsic excitability of neurons that lead to the generation of spontaneous seizures (4). The extent of such a network or region varies, but the area of brain that is indispensable for the generation of seizures (the epileptogenic zone) is often restricted to a discrete focus (5).

Although surgical removal of the epileptogenic zone is commonly used for treatment of mesial temporal lobe epilepsy, it is less successful in focal neocortical epilepsy, often because proximity to eloquent cortex prevents or restricts the extent of resection $(6,7)$. Alternative treatments, such as vagal, cortical, or subcortical stimulation; callosotomy; or ketogenic diet, have met with limited success (8-10). Many patients continue to have poorly controlled epilepsy, with significant effects on their health and welfare $(11,12)$.

Gene therapy targeted to the epileptogenic zone could potentially alleviate seizures by modifying the activity of neurons at the seizure focus without permanently excising brain regions or transecting pathways. Such therapy could, in principle, be used after a brain insult (such as a head injury or status epilepticus) to prevent the development of epilepsy (epileptogenesis) or to stop seizures after epilepsy has been

${ }^{1}$ UCL Institute of Neurology, London WC1N 3BG, UK. ${ }^{2}$ UCL Division of Infection and Immunity, London WC1E 6BT, UK. 3Department of Bioengineering, Stanford University, Stanford, CA 94305, USA. ${ }^{4}$ Open Source Instruments Inc., Watertown, MA 02472, USA. *These authors contributed equally to this work.

†To whom correspondence should be addressed. E-mail: m.walker@ucl.ac.uk (M.C.W.); s.schorge@ucl.ac.uk (S.S.); d.kullmann@ucl.ac.uk (D.M.K.) established. Although eliminating established epilepsy is clinically relevant to a larger target population, previous studies of gene therapy have concentrated on preventing or retarding epileptogenesis after status epilepticus, or providing proof of principle of an increased threshold for provoked seizures (13-19). One study has shown an effect of gene therapy on progression of chronic epilepsy (20). Moreover, these studies have been restricted to experimental models of limbic epilepsy or seizures.

We have used a model of chronic refractory focal neocortical epilepsy in an eloquent region of the cortex (motor cortex) and developed a fully automated electroencephalographic (EEG) seizure detection program to measure the efficacies of three gene therapy strategies on preventing the evolution of spontaneous seizures, reducing seizure activity acutely, and treating established epilepsy.

\section{RESULTS}

\section{Tetanus toxin injection produces a robust, long-lasting} epileptic focus

We optimized a rat model of refractory neocortical epilepsy $(21,22)$. Tetanus toxin injection into the motor cortex is followed by frequent bursts of epileptiform EEG activity associated with clonic movements of the face and forelimb. Similar to human epilepsia partialis continua (23), this seizure activity responds poorly to antiepileptic drugs (22). We recorded the development of epileptiform activity in the EEG using a subdural electrode implanted near the injection site and connected to a wireless subcutaneous transmitter able to sample the EEG at up to $160 \mathrm{~Hz}$ continuously for several weeks (24). Within 72 hours of tetanus toxin injection (10 to $35 \mathrm{ng}$ ), we observed bursts of highfrequency EEG activity, some of which were associated with focal and secondary generalized seizures (Fig. 1A). The EEG of toxin-injected 
A

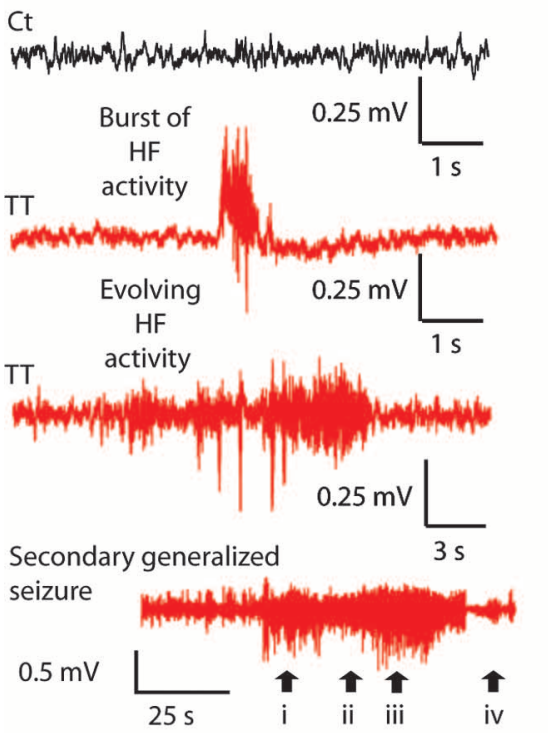

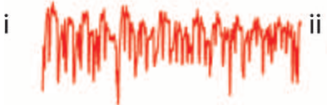

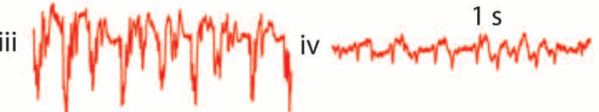

B

C
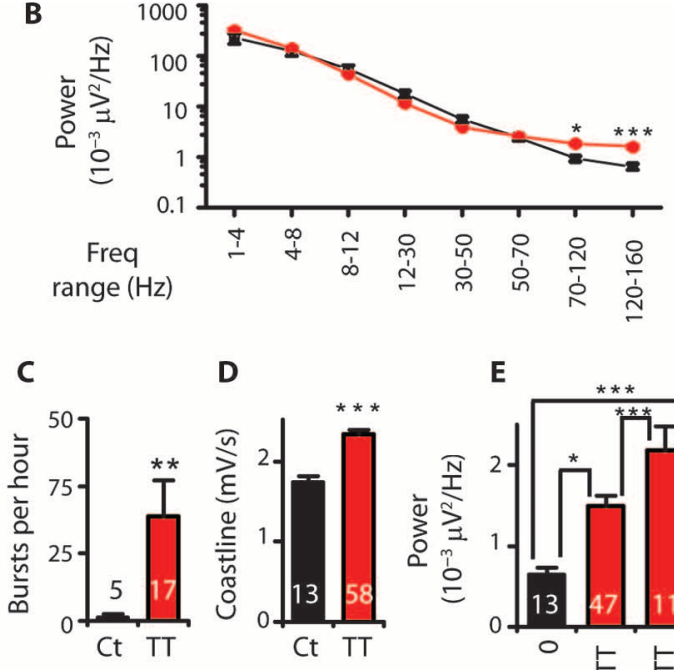

D
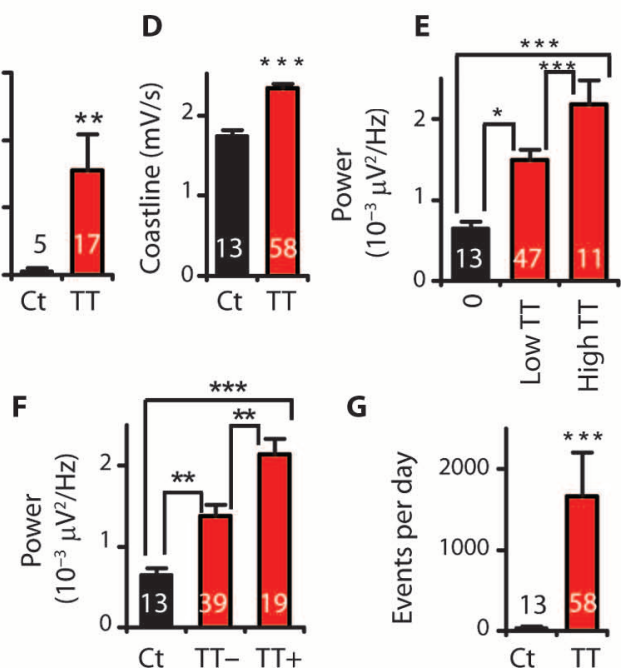

Fig. 1. Tetanus toxin injection induces robust changes in EEG activity and neuronal excitability. (A) Representative control EEG (Ct, black) and EEG after tetanus toxin injection ( $T$, red), and EEG recorded during a secondary generalized seizure (below, red: i, onset; ii, evolution; iii, clonic phase; and iv, postictal period). (B) EEG power 6 to 7 days after tetanus toxin injection (Mann-Whitney: 70 to $120 \mathrm{~Hz}, P=$ 0.024; 120 to $160 \mathrm{~Hz}, P=0.001$; $T$ dose: mean, $14.2 \mathrm{ng}$; range, 10 to $35 \mathrm{ng} ; n=58 ; \mathrm{Ct}: n=13$ ). (C) Number of bursts in 1 hour in Ct and toxin-injected animals (Mann-Whitney $P=0.01$ ). (D) Coastline length (24-hour average; $P=0.001)$. (E) High-frequency power $(120$ to $160 \mathrm{~Hz})$ in $\mathrm{Ct}$ and after low and high toxin dose (low: 10 to $15 \mathrm{ng}$; high: 17.5 to $35 \mathrm{ng}$; Kruskal-Wallis $P=0.0001$; Dunn's multiple comparisons: $\mathrm{Ct}$ versus low, $P<0.05$; low versus high, $P<0.001$ ). (F) High-frequency power in $\mathrm{Ct}$ and in animals without (TT-) or with overt abnormalities (TT+; fig. S3) (Kruskal-Wallis $P<0.0001$; Dunn's multiple comparisons: Ct versus $\Pi-, P<0.01 ; \Pi$ - versus $\Pi+, P<0.01)$. (G) Automatically detected epileptiform events in a 24-hour period (Mann-Whitney $P<0.0001$ ). ${ }^{*} P<0.05 ;{ }^{* *} P<0.01 ;{ }^{* * *} P<0.001$.

animals also exhibited increased power in the 70 - to $160-\mathrm{Hz}$ frequency range (Fig. 1B), which overlaps with the frequency range (100 to $500 \mathrm{~Hz})$ of bursts observed in human neocortical epilepsy (25). The number of high-frequency bursts counted by an observer blinded to the treatment was increased in toxin-injected animals compared to control animals (Fig. 1C).

The EEG coastline length [the cumulative absolute difference in voltage between consecutive data points (26)] was also increased in toxin-injected animals (Fig. 1D) and was strongly correlated with high-frequency power and observer-counted bursts (figs. S1 and S2). In association with increased high-frequency power, some injected animals displayed contralateral forelimb clonus and tonic posturing (fig. S3). Both burst frequency and clinical seizure severity were correlated with tetanus toxin dose (Fig. 1, E and F).

\section{Automated event detection can track epileptogenesis}

Because neither high-frequency power nor coastline length is specific for seizures, we developed a complementary method, which relied on
G

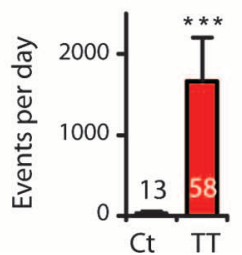

the detection of sudden increases in activity from a recent baseline. The features of such events were automatically compared to a library of EEG patterns, which was generated by a supervised learning algorithm that used concurrent video to identify increases that genuinely corresponded to seizures. In this way, we could distinguish electrographic seizure activity from EEG patterns related to normal behavior such as eating and grooming (fig. S4). Our automated event classification method uses baseline tracking to compensate for slow trends in EEG power that can occur with electrode drift caused by skull thickening with age. The number of epileptiform events thus detected was greatly increased in tetanus toxin-injected animals (Fig. 1G).

\section{Epileptogenesis is associated with increased intrinsic neuronal excitability}

Epileptic activity peaked around 7 days after tetanus toxin injection (see below) and remained elevated for several weeks, suggesting that our model was robust and amenable to quantitative assessment and persists long enough to test gene therapy. The time course further implies that there is a long-lasting alteration in circuit or neuronal excitability that outlives the direct effect of the toxin (27-29). To assess this, we looked for changes in intrinsic neuronal excitability in patch-clamp recordings from motor cortex in ex vivo slices obtained 1 to 4 weeks after 12.5 -ng tetanus toxin injection. To minimize variability attributable to cell type, we focused on adapting (type 2) layer 5 pyramidal neurons (30) (fig. S5). In comparison to control neurons, these cells exhibited a depolarized resting membrane potential (Fig. 2A and fig. S6), an increased input resistance (Fig. 2B), a decreased current threshold for evoking action potentials (Fig. 2C), and an increased likelihood of rebound firing after membrane hyperpolarization (Fig. 2D). They also exhibited a prolonged afterdepolarization potential after injection of a 0.3-ms depolarizing current at threshold for eliciting an action potential, and this sometimes resulted in burst firing (Fig. 2, E to G). Tetanus toxin thus induces a long-lasting increase in the intrinsic excitability of neurons in the epileptic focus. These changes are qualitatively similar to intrinsic excitability alterations in other models of epilepsy (31).

\section{Optogenetic inhibition of a subset of neurons acutely suppresses epileptic activity}

We tested whether acute inhibition of a subset of principal neurons in the focus could reduce network excitability sufficiently to attenuate electrographic seizure activity. Photoactivation of the chloride pump halorhodopsin from Natronomonas pharaonis (NpHR) suppresses 

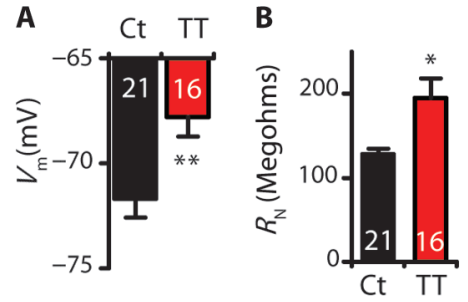

C

E

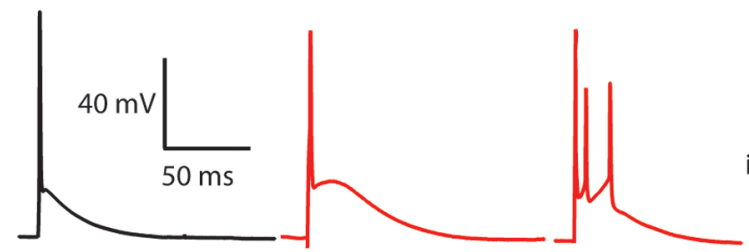

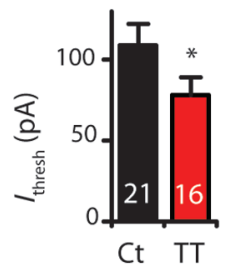

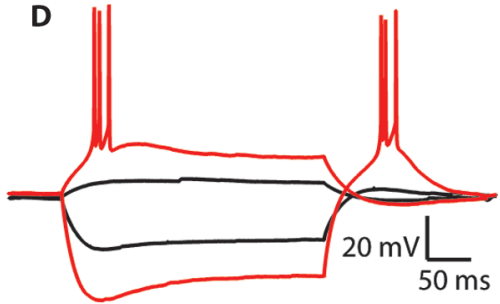

$\mathbf{F}$

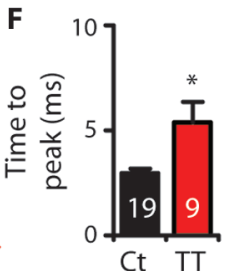

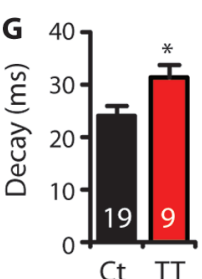

Fig. 2. Injection of tetanus toxin increases excitability of adapting layer 5 pyramidal neurons. (A to C) Neurons in toxin-injected animals $(T T)$ were assessed for $(A)$ resting membrane potential $\left(V_{\text {mi }}\right.$ MannWhitney $P=0.01)$, (B) input resistance $\left(R_{N} ; P=0.02\right)$, and $(C)$ current threshold ( $\left(I_{\text {thresh }} ; P=0.04\right)$. (D) Representative traces from Ct and $\Pi \pi$ neurons evoked by -200 - and +50 -pA current injections. $T$ neurons often showed rebound action potentials ( 7 of 16 versus 0 of 21 in Ct neurons, Fisher's exact test $P=0.0011$ ). When the hyperpolarizing current was adjusted to reach the same voltage as in $\mathrm{Ct}$ neurons, 4 of $16 \pi$ neurons showed rebound action potentials $(P<0.05)$. (E) Sample traces showing response to $0.3-\mathrm{ms}$ depolarizing current injection sufficient to evoke an action potential. This elicited two or more action potentials in 5 of $14 \pi$ neurons and 0 of $19 \mathrm{Ct}$ neurons $(P=0.0084)$. ( $\mathbf{F}$ and $\mathbf{G})$ Afterdepolarization parameters in neurons firing only once, showing longer time to peak $(P=0.04)(\mathrm{F})$ and decay time constant $(P=0.04)(G)$ in tetanus toxin-treated animals. ${ }^{*} P<0.05 ;{ }^{* *} P<0.01$.

burst firing in organotypic hippocampal cultures (32). We injected 12.5 to $17.5 \mathrm{ng}$ of tetanus toxin into the motor cortex together with $1.25 \mu \mathrm{l}$ of high-titer lentivirus carrying NpHR 2.0 tagged with enhanced yellow fluorescent protein (EYFP) under control of a Camk2a promoter (33). We used two sets of control animals: one group was injected with NpHR lentivirus alone; the other received tetanus toxin either with a lentivirus expressing only green fluorescent protein (GFP) or with fluorescent beads. Immediately after the injection, a cannula was implanted at the same site with a $200-\mu \mathrm{m}$-diameter optic fiber (Fig. 3A). Seven to 10 days later, a 561-nm laser was connected to the cannula via a fiberoptic patch cable. We used a 20 -s on/20-s off duty cycle for stimulation to allow $\mathrm{NpHR}$ to recover from desensitization and to minimize the potential disinhibitory effect of depolarization of the $\gamma$-aminobutyric acid type $A\left(\mathrm{GABA}_{\mathrm{A}}\right)$ reversal potential caused by intracellular $\mathrm{Cl}^{-}$ accumulation (34). All experiments and analyses were performed with the experimenter blinded to the treatment. The EEG and behavior were monitored for an initial 1000-s baseline period, followed by 1000 -s intermittent illumination, and then another 1000 -s period after stopping illumination. The animals were subsequently sacrificed, and the brains were examined immunohistochemically to confirm transduction of principal neurons (Fig. 3B).

The behavior of the animals was not visibly affected. Laser illumination in animals coinjected with tetanus toxin and NpHR reduced epileptiform EEG activity (Fig. 3C). Quantification revealed that illumination significantly reduced high-frequency power (Fig. 3D) and EEG coastline (Fig. 3E) compared to the 1000-s baseline period. Lowerfrequency $(<70 \mathrm{~Hz})$ EEG power was not affected. Finally, the frequency of automatically detected epileptiform events was significantly reduced by illumination (Fig. 3F). In control animals injected with NpHR alone, high-frequency power and coastline were lower than in those with crease neuronal excitability in a manner qualitatively similar to what we observed in the tetanus toxin model, including hyperpolarization of the action potential threshold and increased firing of pyramidal neurons (35-37). In contrast, lentiviral overexpression of Kv1.1 in cultured neurons increases the current threshold for action potential generation and reduces the probability of neurotransmitter release (38). We therefore asked whether lentiviral overexpression of Kv1.1 in vivo also attenuated the excitability of layer 5 pyramidal neurons in acute brain slices.

Injection of a lentivirus expressing Kv1.1 under a cytomegalovirus promoter, with a GFP reporter, led to local transduction of layer 5 neurons of the motor cortex (Fig. 4A and fig. S7). Because Kv1.1 overexpression in interneurons could potentially disinhibit the circuit, we investigated the cell type specificity of transduction. Co-staining for markers of glutamatergic, GABAergic, and glial cells [CaMKII $\alpha$, GAD67, and GFAP (glial fibrillary acidic protein), respectively] revealed preferential transduction of excitatory neurons, with minimal expression of GFP in interneurons and no detectable expression in glia (fig. S8). Recordings from GFP-positive layer 5 pyramidal neurons in ex vivo cortical slices (fig. S9) showed that subthreshold properties including resting membrane potential and input resistance were largely unaffected by Kv1.1 overexpression (Fig. 4, B and C). In contrast, Kv1.1-transduced neurons required more depolarization to fire action potentials, in particular trains (Fig. 4, D and E), than either untransduced neurons or neurons transduced with a control lentivirus expressing GFP alone. In addition, the relationship of firing frequency to current injection was shifted, with similar currents eliciting fewer action potentials in Kv1.1-overexpressing neurons (Fig. 4, F and G). Combined, these effects would be more likely to attenuate than silence completely neuronal and network activity. We observed no behavioral deficits in animals overexpressing Kv1.1 in their primary motor cortex, monitored for 4 weeks after Kv1.1-GFP lentivirus 
A

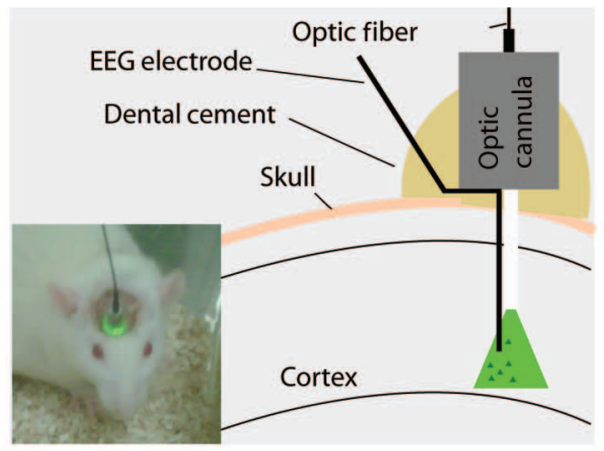

B

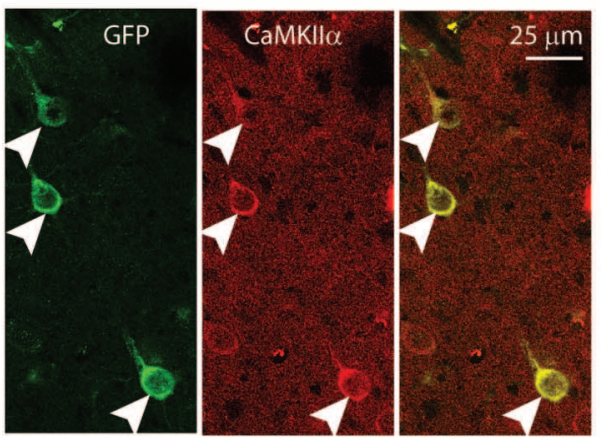

C

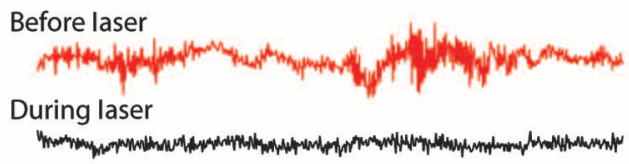

After laser
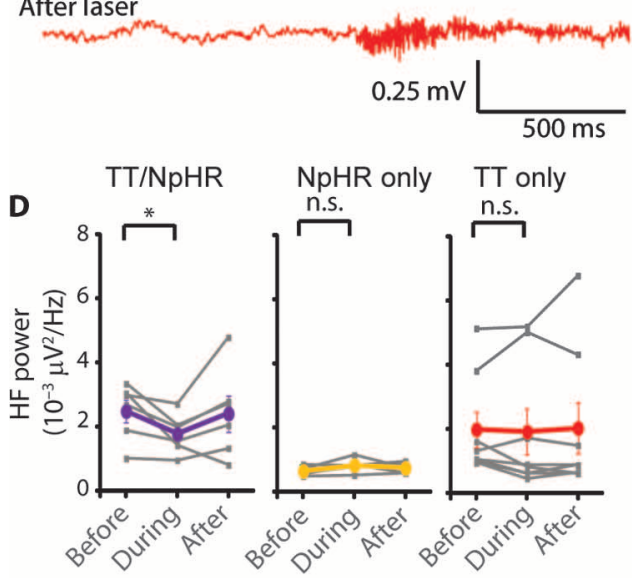

E

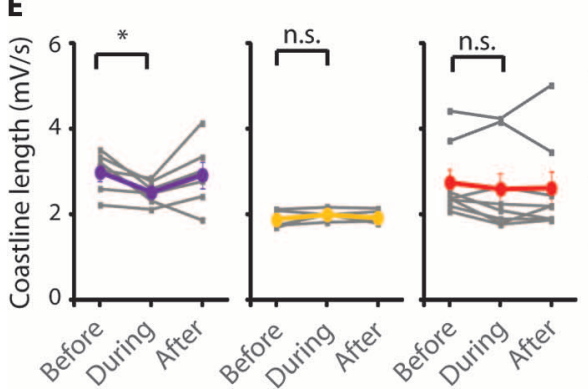

Fig. 3. Acute optogenetic attenuation of epileptic activity. (A) Schematic of the implanted headstage for simultaneous EEG recording and optical stimulation. (B) Immunohistochemical staining of cells in the focus showing GFP-containing transduced neurons (left) and CaMKIl $\alpha$ staining of the same neurons (middle). Right, merged images. (C) Representative EEG traces before, during, and after 561-nm laser illumination. (D) Mean high-frequency (HF) power in animals injected with tetanus toxin (TT) and NpHR lentivirus (left, $n=6$ ), showing a significant decrease upon laser stimulation ( $n=6$; Wilcoxon matched pairs signed-rank test, $P=0.03$; gray, individual experiments; purple, mean \pm SEM). Baseline high-frequency power was lower in animals injected with NpHR lentivirus alone (middle; $n=5$; unpaired two-tailed $t$ test, $P<0.001$ ) and unaffected by laser illumination (yellow, mean \pm SEM). Laser illumination had no effect on high-frequency power in control animals that were injected with TT together with either GFPexpressing control virus or fluorescent beads (right; $n=8$; red, mean $\pm \mathrm{SEM}$ ). n.s., not significant. (E) Mean coastline length, plotted as in (D), was decreased by illumination in animals injected with TT and NpHR (Wilcoxon matched pairs signed-rank test, $P=0.03$ ) but not in control animals injected with TT and GFP. (F) Automatically detected epileptiform events in the same animals, plotted as in (D), were decreased upon laser illumination (paired $t$ test after log transform, $P=0.048$ ). Event counts in animals injected with TT together with GFP or beads were unaffected by laser illumination (before, $50 \pm 25$; during, $47 \pm 30$; after, $51 \pm 40) .{ }^{*} P<0.05$.

injection (fig. S10). When sacrificed at 1 or 4 weeks after injection, we observed no change in the neuronal marker NeuN and no increase in GFAP other than that caused by the injection needle (fig. S11).

Overexpression of Kv1.1 can block the development of epilepsy We next tested whether overexpression of Kv1.1 was sufficient to disrupt the development of epilepsy. To test this, we coinjected Kv1.1 lentivirus together with $12.5 \mathrm{ng}$ of tetanus toxin and compared the different measures of epileptic activity in the EEG. Coinjection of Kv1.1 with tetanus toxin completely prevented the toxin-induced increase in both highfrequency EEG power (Fig. 5A) and coastline (Fig. 5, B and C) when compared to animals receiving the same dose of tetanus toxin without Kv1.1. Kv1.1 also almost completely prevented the occurrence of automatically detected epileptiform events after tetanus toxin treatment (Fig. 5D). These data suggest that, as with halorhodopsin, transduction of only a subset of neurons was sufficient to have robust effects on network activity.

Gene therapy with Kv1.1 can reverse established epilepsy Can overexpression of Kv1.1 be used to suppress seizure activity after the epileptic state has become established? To answer this question, we injected either Kv1.1 lentivirus or GFP-only lentivirus into the seizure focus 1 week after the 12.5-ng tetanus toxin injection and continued to record the EEG for several weeks. Compared to animals injected with GFP, animals receiving the Kv1.1 lentivirus injection showed a reduced coastline (Fig. 6, A and B) and high-frequency power (Fig. 6C). We also noted a significant difference of effect of treatment on the number of epileptiform events between animals injected with Kv1.1 lentivirus and those injected with GFP alone (Fig. 6D). Indeed, the epileptiform event frequency returned to baseline after 4 weeks in Kv1.1-treated animals but remained elevated in animals receiving GFP.

\section{DISCUSSION}

This study shows that spontaneous electrographic seizure activity can be attenuated acutely with an optogenetic strategy and that Kv1.1 gene 

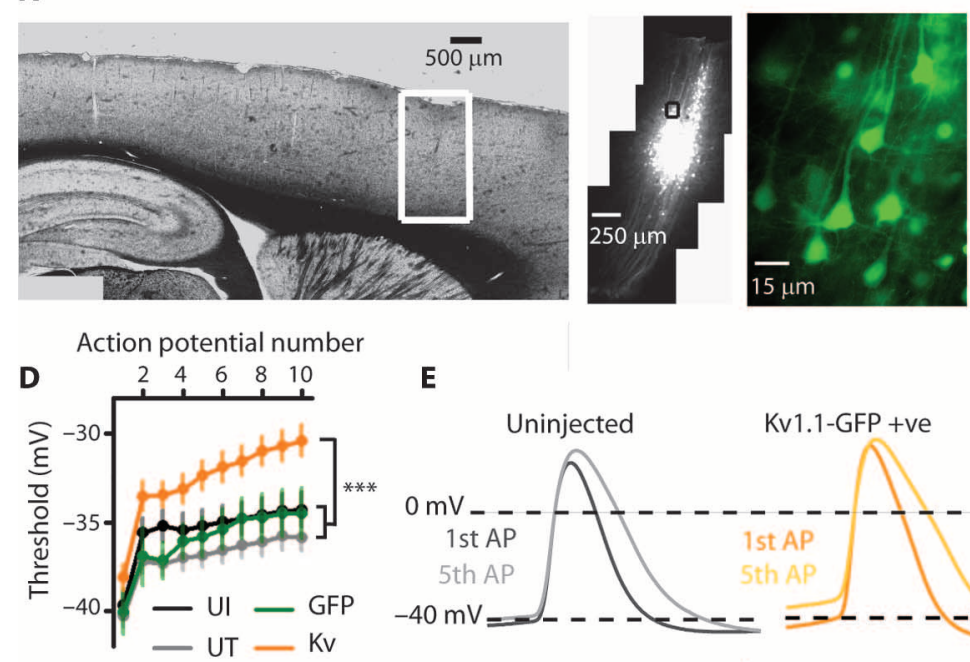

E
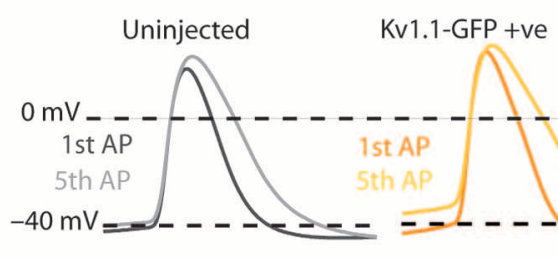

Kv1.1-GFP +ve

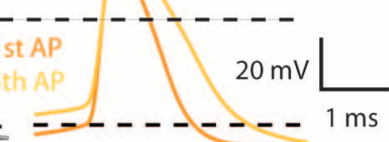

G

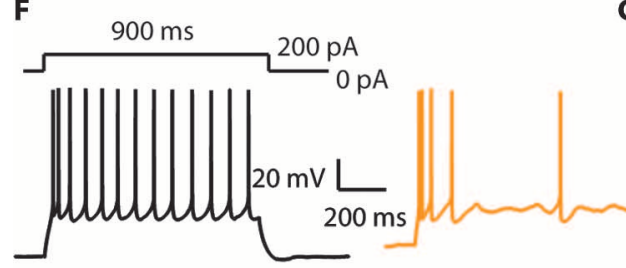

\section{송}

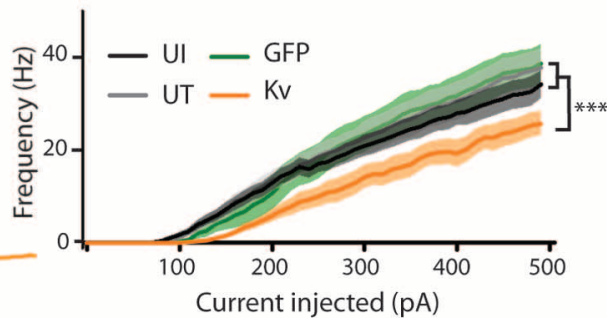

Fig. 4. In vivo injection of Kv1.1 lentivirus attenuates excitability of adapting layer 5 pyramidal neurons. (A) GFP expression in a restricted area of motor cortex after injection of Kv1.1-GFP lentivirus. (B and C) Subthreshold properties in neurons from uninjected animals (UI) and animals injected with GFPonly lentivirus (G), and in GFP-negative untransduced (UT) and neighboring GFP-positive (Kv) neurons from Kv1.1-GFP-injected animals [resting membrane potential (RMP) (B) and resting input resistance $\left.\left(R_{N}\right)(C)\right]$. (D to $\left.\mathbf{G}\right)$ Neuronal excitability in neurons overexpressing Kv1.1. (D) Action potential threshold in a train of spikes $(P<0.0005$ for an effect of group, linear mixed model analysis). (E) First and fifth action potentials showing depolarized threshold but no difference in accommodation (spike amplitude, rise time, decay, and width at half-maximum). (F) Representative traces from neighboring untransduced (black) and Kv1.1-overexpressing (orange) neurons in response to $+200-p A$ current injection. (G) Frequency-current relationship showing a significant decrease in firing rate in neurons overexpressing Kv1.1 $(P<0.0001$ for difference between groups, log-linear mixed model). All recordings at $36 \pm 1{ }^{\circ} \mathrm{C}, 7$ to 20 days after virus injections. ${ }^{* *} P<0.001$.

therapy both prevents the evolution of spontaneous seizures after an epileptogenic insult and has a robust therapeutic effect on established focal neocortical epilepsy.

Many experimental studies of antiepileptic treatments have used rodent models of seizures precipitated acutely by electrical stimulation or chemoconvulsants. These are, however, indirectly related to human epilepsy in which neuronal and network alterations lead to the occurrence of spontaneous seizures, and treatment trials in these models have translated poorly (39). Chronic models of limbic epilepsy have the advantages that seizures occur spontaneously and that they resemble human epilepsy associated with hippocampal pathology. Although the epileptogenic zone often involves a distributed network, partial surgical resection of the temporal lobe can often prevent seizures in human epilepsy associated with hippocampal sclerosis. We have focused instead on a model of focal neocortical epilepsy that recapitulates features of epilepsia partialis continua (23). This is a particularly severe and drug-resistant form of epilepsy characterized by the occur-
B UI G UT Kv
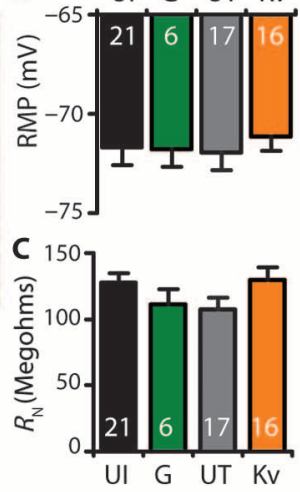

rence of continuous or almost continuous motor seizure activity, for which surgical resection is often contraindicated by unacceptable risk to motor function. We took advantage of the tetanus toxin model because it leads to a stable state characterized by EEG features similar to those seen in chronic human focal neocortical epilepsy (unlike some rodent neocortical seizure models in which seizures occur only transiently). Both in the human condition and in the animal model, bursts of highfrequency EEG activity are associated with a motor manifestation $(22,23)$; however, to minimize mortality and animal suffering, we mainly used a low dose of tetanus toxin, which yielded clear EEG changes but only relatively infrequent overt motor seizures. Although we have shown that seizure activity in EEG recordings can be reduced with lentiviral gene therapy in this model, it remains to be determined whether epilepsies with larger or more widely distributed epileptogenic zones are amenable to such therapies.

Optogenetic inhibition of pyramidal neurons was sufficient to acutely attenuate seizure activity in the EEG, underlining the potential efficacy of a strategy that targets a neuronal subtype in a discrete area of the brain, which cannot be achieved with systemic antiepileptic drugs. Although this optogenetic approach is invasive, because of the need to deliver light to the transduced neurons, it could, in principle, allow seizure suppression on demand. High-frequency EEG oscillations build up before neocortical seizure onset and occur preferentially in epileptogenic areas $(40,41)$, suggesting that automated detection of high-frequency oscillations could be used to trigger illumination via a laser or light-emitting diodes, with potential advantages over electrical stimulation (8), focal cooling (42), or targeted drug delivery (43). This approach offers the prospect of a device analogous to an implantable defibrillator to stop seizures without permanent alteration of neuronal properties.

For long-term treatment, we focused on Kv1.1 overexpression, which was well tolerated and suppressed electrographic seizure activity for at least 4 weeks. Although it was technically difficult to record the EEG for longer periods, local expression persisted at least 6 months in vivo (fig. S12), suggesting that the effects of Kv1.1 expression are likely to persist beyond 4 weeks. Thus far, we have only examined one focal epilepsy model, but the data indicate that gene therapy using Kv1.1 is potentially curative. We have previously shown that Kv1.1 overexpression reduces not only neuronal excitability but also neurotransmitter release from the terminals of transduced neurons in culture (38), consistent with expression in both somatodendritic and axonal compartment. This dual mode of action is potentially advantageous over 
A

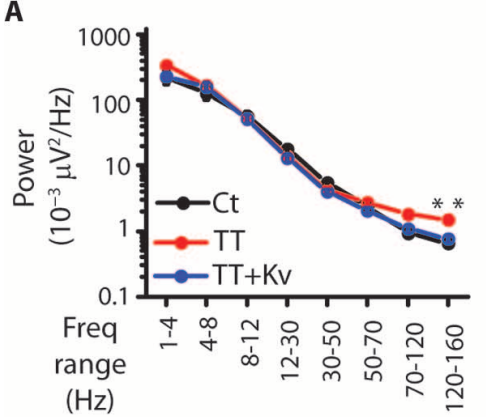

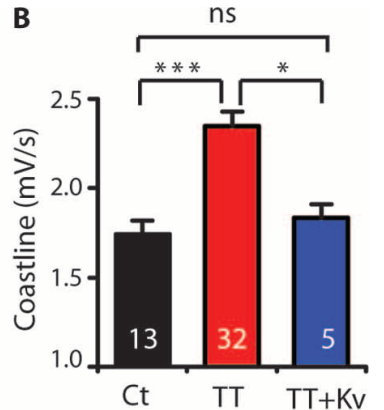

C

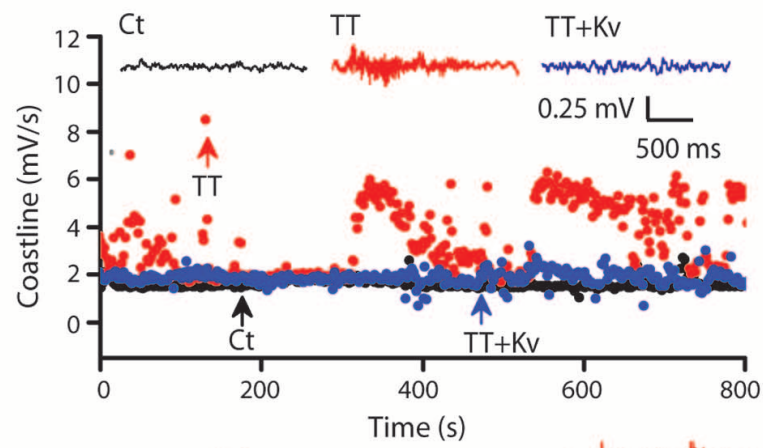

D

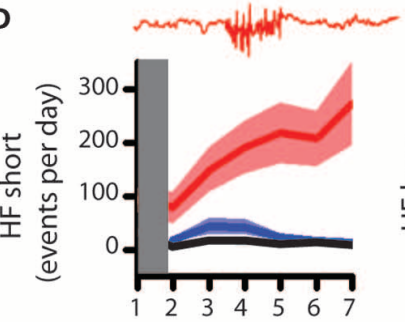

Days post-injection
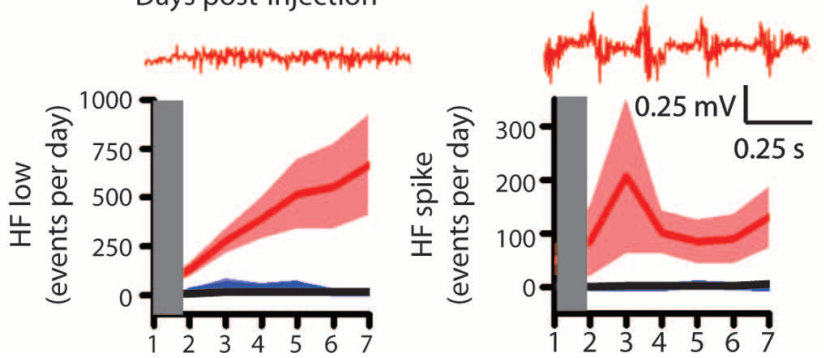

Fig. 5. Kv1.1 lentivirus injection with tetanus toxin prevents focal neocortical epileptogenesis. (A) Effect of coinjection of Kv1.1-GFP lentivirus with tetanus toxin ( $T T+K v)$ on high-frequency power (EEG analyzed over 24 hours, 7 days after injection; Kruskal-Wallis $P<0.0001$, SEM concealed by symbols). (B) Effect of coinjection of Kv1.1 and tetanus toxin on EEG coastline $(P<0.001)$. (C) Sample coastline analysis from $800 \mathrm{~s}$ of EEG recorded from three representative animals 7 days after injection (each point represents the coastline length of a 2-s segment of EEG); arrows indicate expanded EEG traces (top): Ct (black), control; TT (red), TT-injected animal; TT+Kv (blue), TT + Kv1.1-injected animal. (D) Effect of Kv1.1 and tetanus toxin coinjection on epileptiform events measured by automated event classification. Four categories of events are shown with representative library templates (traces, see also fig. S4; HF short, HF long, HF low, and HF spike defined in fig. S4). Animals that received Kv1.1 had fewer epileptiform events than those that received tetanus toxin only (all events aggregated, log-linear mixed model $P<0.001$ ). Kv1.1 injection reduced epileptiform events to rates indistinguishable from $\mathrm{Ct}$ animals that did not receive tetanus toxin $(P=0.511)$. Data are means \pm SEM. Gray area indicates quarantine period after virus injection. ${ }^{*} P<0.05 ;{ }^{* *} P<0.01 ;{ }^{* * *} P<0.001$.
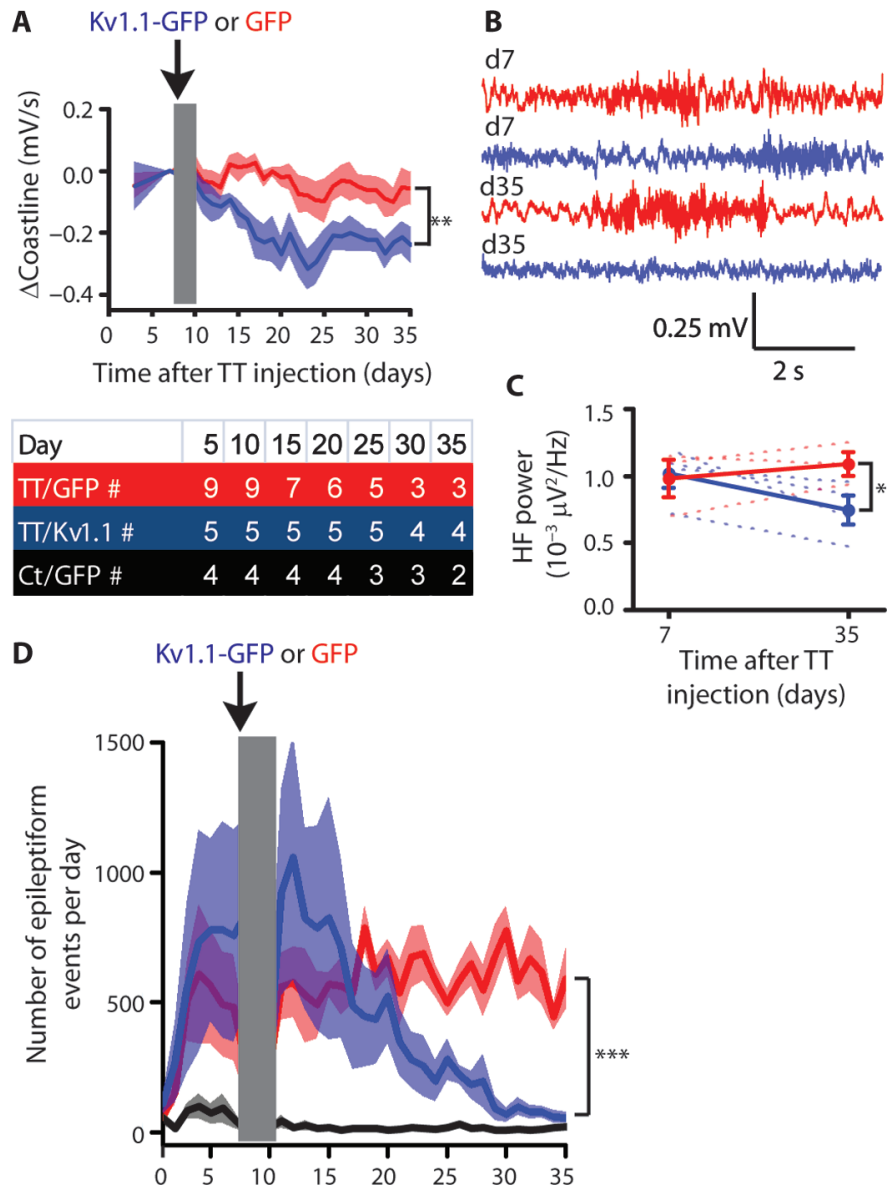

Fig. 6. Kv1.1 lentivirus treats focal neocortical epilepsy. (A) EEG coastline in tetanus toxin-treated animals relative to the value at day 7 , just before delayed injection (arrow) of Kv1.1-GFP lentivirus (blue) or GFP-only lentivirus (red). Gray bar indicates quarantine period (linear mixed model, $P=$ 0.004). Below: Numbers of animals at different time points (another control group without tetanus toxin but injected with GFP lentivirus exhibited no change in coastline length). (B) Sample EEG traces from two animals at days 7 and 35 (red, GFP; blue, Kv1.1-GFP). (C) Effect of Kv1.1 injection after established epileptogenesis on high-frequency power (unpaired $t$ test for change in power versus GFP, $P<0.05$ ). (D) Effect of Kv1.1 injection after established epileptogenesis on the number of epileptiform events (loglinear mixed model for treatment with Kv1.1 versus GFP, $P<0.0001)$. Red, tetanus toxin followed by GFP; blue, tetanus toxin followed by Kv1.1; black, GFP only. ${ }^{*} P<0.05 ;{ }^{* *} P<0.01 ;{ }^{* *} P<0.001$.

other strategies to silence neurons (44): If synaptic inputs to transduced neurons undergo a homeostatic compensation for their reduced excitability (45), neurotransmitter release from their terminals should remain attenuated.

Overexpression of Kv1.1 was effective not only in preventing the development of epilepsy but also in progressively reducing seizure activity after epilepsy was established. This suggests that Kv1.1 overexpression is both antiepileptogenic and antiepileptic. However, lentiviral treatment blurs the distinction between antiepileptic and anticonvulsant effects because the decrease in EEG activity was recorded during continued Kv1.1 overexpression. It will be important to determine whether conditional expression of Kv1.1 during a finite period could achieve 
similar effects or whether sustained overexpression of Kv1.1 is required to maintain seizure suppression.

The primary action of tetanus toxin is to cleave the SNARE (soluble $\mathrm{N}$-ethylmaleimide-sensitive factor attachment protein receptor) protein synaptobrevin (46), and the depolarization, lowered threshold, and predisposition to burst firing seen in pyramidal neurons from toxininjected animals are likely to be consequences of altered transcription of multiple channels. Similar increases in intrinsic excitability have been observed in several other epilepsy models (31), with evidence for changes in T-type $\mathrm{Ca}^{2+}$ channels (47), hyperpolarization-activated conductance $(48)$, and A-type $\mathrm{K}^{+}$channels $(49,50)$ [see also $(51,52)$ ]. Enhanced intrinsic excitability has also been reported in models of neuropathic pain $(53,54)$. Kv1.1 overexpression may thus be effective in other disorders characterized by excessive neuronal firing as well as in a wide range of epilepsy etiologies.

The strategy proposed here for treatment of focal epilepsy does not preclude surgical resection of the epileptogenic zone. Instead, local gene therapy potentially offers a less invasive treatment, which, if unsuccessful, could be followed by definitive resection. However, where ablative surgery is contraindicated by proximity to eloquent cortex or critical white matter tracts, we anticipate that the cell type selectivity and graded effects of gene therapy may make it an attractive option.

\section{MATERIALS AND METHODS}

\section{Lentivirus production}

For NpHR expression, vesicular stomatitis virus glycoprotein (VSVG)pseudotyped lentivirus tagged with EYFP under the Camk2a promoter (LV-Camk2a-NpHR 2.0-EYFP-WPRE) was produced according to protocols in the laboratory of $\mathrm{M}$. E. Collins [University College London (UCL)]. 293FT cells (Invitrogen) were seeded 24 hours before transfection with the transfer vectors p8.91 (gag/pol expressor) and pMD.G (VSVG expressor) in FuGENE 6 (Roche), Opti-MEM (Invitrogen), and sterile TE buffer (Invitrogen). The lentivirus-containing supernatant was harvested 24, 48, and 72 hours after transfection and centrifuged at $20,000 \mathrm{rpm}$ for 2 hours at $4^{\circ} \mathrm{C}$, and the virus was then resuspended, aliquoted, and stored long term at $-80^{\circ} \mathrm{C}$. The concentrated viral titer was determined by quantitative polymerase chain reaction to be $10^{8}$ infectious units (IU)/ml. High-titer lentivirus (Kv1.1-GFP: pCDH1CMV-KCNA1-EF1-copGFP, $2.6 \times 10^{9} \mathrm{IU} / \mathrm{ml}$, or GFP only: pCDH1CMV-MCS-EF1-copGFP, $1.1 \times 10^{9} \mathrm{IU} / \mathrm{ml}$ ) was obtained from System Biosciences. The human Kv1.1-GFP lentiviral construct was as described in (38). In a subset of animals studied at 4 months, we estimated that neurons were transduced within a volume of about $0.04 \mathrm{~mm}^{3}$ (NpHR lentivirus) or $0.14 \mathrm{~mm}^{3}$ (Kv1.1 lentivirus).

\section{Tetanus toxin and surgery}

Male Sprague-Dawley rats (6 to 12 weeks old) were anesthetized with isoflurane and placed in a stereotaxic frame (Kopf). Tetanus toxin (gift of G. Schiavo) was coinjected with lentivirus encoding Kv1.1-GFP, $\mathrm{NpHR}$, or GFP only or with fluorescent beads (FluoSpheres, $10 \mu \mathrm{m}$, Invitrogen) in a final volume of 1.0 to $1.5 \mu \mathrm{l}$ into layer 5 of the right motor cortex (coordinates, $2.4 \mathrm{~mm}$ lateral and 0 to $1.0 \mathrm{~mm}$ anterior of bregma at a depth of $1.0 \mathrm{~mm}$ from pia). Except where indicated, $12.5 \mathrm{ng}$ of tetanus toxin was injected. An EEG transmitter [A3019D, Open Source Instruments (24)] was implanted subcutaneously with a subdural intracranial recording electrode positioned above the injection site. A reference electrode was implanted in the contralateral skull. For sequential injections, a cannula (Plastics One) was implanted above the tetanus toxin injection site, and 7 days later, lentivirus was administered via the cannula. For optogenetic experiments, an optic fiber [core diameter, $200 \mu \mathrm{m}$; numerical aperture (NA), 0.22; cannula length, 1.5 to $1.8 \mathrm{~mm}$; Doric Lenses] was implanted above the injection site. Animals were housed separately in Faraday cages, and EEG was recorded continuously for up to 6 weeks after surgery. Animal experiments were conducted in accordance with the Animals (Scientific Procedures) Act 1986.

\section{EEG analysis}

EEG was processed with the Neuroarchiver tool (Open Source Instruments), which determined EEG power for different frequency bands. EEG epochs were also exported into LabVIEW (National Instruments) for coastline analysis. Glitches were excluded if they exceeded $5 \times$ root mean square, and the signal was high pass-filtered at $10 \mathrm{~Hz}$ before calculating the coastline as the sum of the absolute difference between successive points. Burst, coastline, and EEG high-frequency power analysis were averaged over 24 hours.

\section{Automated epileptiform event counting}

Event sorting is explained in fig. S4. A more complete description of the seizure detection algorithm with source code is available at http:// www.opensourceinstruments.com/Electronics/A3018/Seizure_Detection. html\#Similarity\%20of\%20Events.

\section{Optogenetic stimulation in vivo}

Optogenetic studies were performed on days 7 to 10 after surgery. The implanted optic cannula was connected to a 561-nm laser (CrystaLaser) via a fiber-optic patch cord (NA, 0.22; Doric Lenses) and a rotating commutator (Doric Lenses). The laser output was calibrated with a digital optical power meter (PM100D, Thorlabs), aiming for an intensity of $25 \mathrm{~mW}$ at the implantable fiber tip, corresponding to an irradiance of about $22 \mathrm{~mW} / \mathrm{mm}^{2}$ at a distance of 0.5 to $1 \mathrm{~mm}$, previously reported to be sufficient to inhibit $98 \%$ of spikes in brain slice experiments (33).

\section{Brain slice preparation}

Rats were deeply anesthetized with sodium pentobarbital (intraperitoneally) and transcardially perfused with cold $\left(4^{\circ} \mathrm{C}\right)$ modified artificial cerebrospinal fluid (ACSF) containing high sucrose and low sodium: $85 \mathrm{mM} \mathrm{NaCl}, 2.5 \mathrm{mM} \mathrm{KCl}, 1.25 \mathrm{mM} \mathrm{NaH}_{2} \mathrm{PO}_{4}, 20 \mathrm{mM}$ $\mathrm{NaHCO}_{3}, 10 \mathrm{mM}$ Hepes, $25 \mathrm{mM}$ glucose, $75 \mathrm{mM}$ sucrose, $0.5 \mathrm{mM}$ $\mathrm{CaCl}_{2}, 4 \mathrm{mM} \mathrm{MgCl}, 2 \mathrm{mM}$ kynurenic acid, $\mathrm{pH}$ 7.3, oxygenated with $95 \% \mathrm{O}_{2} / 5 \% \mathrm{CO}_{2}$. The rat was then decapitated, and the brain was rapidly removed into cold sucrose-ACSF. Parasagittal slices $(300 \mu \mathrm{m}$ thick) were prepared with a vibrating slicer (Leica VT 1200S) and transferred to a holding chamber containing modified ACSF at $35^{\circ} \mathrm{C}$. After 20 min, slices were transferred to a holding chamber containing ACSF at room temperature: $125 \mathrm{mM} \mathrm{NaCl}, 2.5 \mathrm{mM} \mathrm{KCl}, 1.25 \mathrm{mM}$ $\mathrm{NaH}_{2} \mathrm{PO}_{4}, 20 \mathrm{mM} \mathrm{NaHCO}, 5 \mathrm{mM}$ Hepes, $25 \mathrm{mM}$ glucose, $2 \mathrm{mM}$ $\mathrm{CaCl}_{2}, 1 \mathrm{mM} \mathrm{MgCl}$, pH 7.3, oxygenated with $95 \% \mathrm{O}_{2} / 5 \% \mathrm{CO}_{2}$. Slices were used for recordings 0.5 to 4 hours after preparation. For recording, slices were transferred to the stage of an upright microscope equipped with epifluorescence (Olympus BX51WI) and constantly perfused with ACSF at $36 \pm 1^{\circ} \mathrm{C}$. 


\section{Electrophysiology}

Somatic whole-cell recordings were obtained under visual control with infrared difference interference contrast. Pipette resistance was 3 to 5 megohms when filled with intracellular solution. Current-clamp recordings were obtained with a MultiClamp 700A amplifier (Molecular Devices) and an intracellular solution containing $135 \mathrm{mM}$ K-gluconate, $4 \mathrm{mM} \mathrm{KCl}, 10 \mathrm{mM}$ Hepes, $4 \mathrm{mM}$ Mg-ATP, $0.3 \mathrm{mM}$ Na-GTP, $10 \mathrm{mM}$ $\mathrm{Na}_{2}$-phosphocreatine, $0.01 \mathrm{mM}$ Alexa Fluor 594, and $0.2 \%(\mathrm{w} / \mathrm{v})$ biocytin ( $\mathrm{pH} 7.3,291$ to $293 \mathrm{mOsm}$ ). Layer 5 pyramidal neurons in the motor cortex were identified by their somatic shape and apical dendrite and characteristic spiking pattern. Neurons from toxin-injected animals were patched within the injection site (identified by visualization of the coinjected fluorescent beads or GFP virus). Neurons expressing either Kv1.1-GFP or GFP only were identified in real time with PatchVision software (Scientifica). Data were filtered at $4 \mathrm{kHz}$ and acquired at $50 \mathrm{kHz}$ with a DigiData 1200 series interface board, with current command routines written in Clampex v9.2 (Molecular Devices).

Subthreshold properties were measured by injecting 300-ms hyperpolarizing or depolarizing currents, whose amplitude was increased in 25-pA increments until an action potential was elicited. Action potential parameters were measured at threshold, and accommodation was measured when the depolarizing current was increased until at least 10 spikes were elicited. Frequency-current relationships were obtained from 900-ms current injections. Afterdepolarization parameters (amplitude, duration, and decay) were measured by injecting a 0.3-ms current whose amplitude was gradually increased until at least one action potential was elicited.

Electrophysiological data were analyzed offline in IGOR Pro v6.0 (WaveMetrics) with routines originally written by J. Waters (Northwestern University) and adapted by I. Oren (UCL) and R.W. (55). Input resistance at rest was estimated from the slope at $0 \mathrm{pA}$ of a quadratic fit to a plot of steady-state voltage against current at the end of a 300-ms current injection, after relaxation of sag potentials. Action potential threshold was defined as the point where $\mathrm{d} V / \mathrm{d} t$ first exceeded $40 \mathrm{mV} / \mathrm{ms}$. The initial interspike interval used to assess adaptation was taken from the time between the thresholds of the second and the third spikes. The decay of the afterdepolarization was estimated from a monoexponential fit to the relaxation of the membrane potential.

\section{Motor behavior}

Rats injected with either Kv1.1-GFP or GFP-only lentivirus into the right motor cortex (volume and coordinates as above) were assessed with a grid-walking test (56). Each animal was placed on a $52 \times 32-\mathrm{cm}$ elevated horizontal platform consisting of a square painted steel wire array (4-cm spacing) and allowed to explore the arena for $2 \mathrm{~min}$. Two observers blinded to the lentiviral treatment independently scored the number of forelimb foot faults (where the entire foot passed through the gap). The test was applied before the lentiviral treatment and then at weekly intervals for 4 weeks.

\section{Immunohistochemistry}

Animals were transcardially perfused under terminal anesthesia (sodium pentobarbital) with heparinized phosphate-buffered saline (PBS) (10 U of heparin/ml of PBS), followed by $4 \%$ paraformaldehyde (PFA)/PBS. Brains were removed and left in 4\% PFA/PBS overnight at $4^{\circ} \mathrm{C}$ and then washed in PBS. Parasagittal and coronal slices (30 to $150 \mu \mathrm{m}$ thickness) were cut on a vibrating slicer or freezing vibratome (both Leica Microsystems) and mounted on microscope slides (Thermo Scientific) with polyvinyl alcohol mounting medium with DABCO antifading reagent (Sigma), Vectashield (Vector Labs), or Mowiol (Polysciences Inc.) mounting medium. Bright-field and fluorescence images were analyzed and assembled with ImageJ software (Mosaic) plugin). For characterization of the expression of $\mathrm{NpHR}$, sections were permeabilized in PBS and $0.2 \%$ Triton X-100 for $10 \mathrm{~min}$ and then blocked with DAKO-blocking medium (Dako) for 1 hour on a shaker. Subsequently, they were incubated for 2 days in primary antibodies against CaMKII $\alpha$ (1:100 to 1:200, Thermo Scientific) and GFP (1:3000, Abcam, ab13970, or 1:800, Aves Labs, GFP-1020). After further washes in PBS, the sections were incubated in secondary antibodies (1:1000, Abcam, and labeled with Alexa Fluor 488, Alexa Fluor 568, or tetramethylrhodamine) overnight at $4^{\circ} \mathrm{C}$. Sections were then incubated in $4^{\prime}$,6-diamidino-2-phenylindole to identify nuclei. For Kv1.1 lentivirusinjected animals, slices were permeabilized in PBS with $0.3 \%$ Triton X-100 (PBST), blocked in 4\% normal goat serum, and incubated overnight in mouse anti-CaMKII $\alpha$ (1:200), mouse anti-GAD67 (1:500), mouse anti-GFAP, or mouse anti-NeuN (1:200 or 1:400, mab05-532, mab5406, mab3402, and mab377, respectively; Millipore) in PBST. After washing in PBS, slices were incubated for 2 hours at room temperature in goat anti-mouse Alexa Fluor 546 (1:1000, Invitrogen). After $3 \times$ 20 -min washes in PBS, the slices were mounted in polyvinyl alcoholDABCO or Vectashield. Images were obtained with a Zeiss LSM 510 or 710 confocal microscope, or a Leica DM5000B upright fluorescence microscope at $\times 10$ magnification and a Leica DM2500 upright confocal microscope at $\times 40$ magnification. The spatial extent of viral expression was estimated by multiplying the slice thickness with the size of the area exhibiting EYFP fluorescence at $\times 10$ magnification, and summing over consecutive slices. Image analysis and cell counting were performed with GIMP and ImageJ.

\section{Statistical analysis}

Paired or unpaired parametric or nonparametric $t$ tests or analysis of variances (ANOVAs) were performed as appropriate with InStat V3 software (GraphPad). For analysis of time series data or repeated measures, we used generalized mixed linear model analysis (SPSS version 12). For counts of epileptiform events after treatment, we used a log-linear model with random effect of day (autoregressive covariance) and fixed effect of the interaction between treatment group and time receiving treatment. For analysis of change in coastline after treatment, we used a linear model with random effect of day (autoregressive covariance) and fixed effect of treatment group. Action potential threshold and frequency (counts) were analyzed with a linear mixed model and a log-linear mixed model, respectively.

\section{SUPPLEMENTARY MATERIALS}

www.sciencetranslationalmedicine.org/cgi/content/full/4/161/161ra152/DC1 Fig. S1. Correlation of EEG coastline length with increases in high-frequency power.

Fig. S2. Correlation of burst counting by a blinded observer with high-frequency power and coastline length.

Fig. S3. Electroclinical features of the tetanus toxin model.

Fig. S4. Automated event classification.

Fig. S5. Criteria used to identify adapting layer 5 pyramidal neurons.

Fig. S6. Biophysical properties of type 2 pyramidal neurons in animals injected tetanus toxin.

Fig. S7. Estimation of volume of tissue transduced with Kv1.1-GFP lentivirus.

Fig. S8. Preferential transduction of excitatory neurons with Kv1.1 lentivirus.

Fig. S9. Identification of layer 5 neurons. 
Fig. S10. Behavioral assessment in animals injected with Kv1.1-GFP lentivirus.

Fig. S11. Immunohistochemical analysis of neuronal and glial markers after Kv1.1-GFP lentivirus injection.

Fig. S12. Stable neuronal transduction with Kv1.1 lentivirus.

\section{REFERENCES AND NOTES}

1. A. K. Ngugi, C. Bottomley, I. Kleinschmidt, J. W. Sander, C. R. Newton, Estimation of the burden of active and life-time epilepsy: A meta-analytic approach. Epilepsia 51, 883-890 (2010).

2. P. Kwan, S. C. Schachter, M. J. Brodie, Drug-resistant epilepsy. N. Engl. J. Med. 365, 919-926 (2011).

3. J. F. Annegers, W. A. Hauser, L. R. Elveback, Remission of seizures and relapse in patients with epilepsy. Epilepsia 20, 729-737 (1979).

4. Jasper's Basic Mechanisms of the Epilepsies-NCBI Bookshelf; http://www.ncbi.nlm.nih gov/books/NBK50785/.

5. F. Rosenow, H. Lüders, Presurgical evaluation of epilepsy. Brain 124, 1683-1700 (2001).

6. S. U. Schuele, H. O. Lüders, Intractable epilepsy: Management and therapeutic alternatives. Lancet Neurol. 7, 514-524 (2008).

7. J. de Tisi, G. S. Bell, J. L. Peacock, A. W. McEvoy, W. F. Harkness, J. W. Sander, J. S. Duncan The long-term outcome of adult epilepsy surgery, patterns of seizure remission, and relapse: A cohort study. Lancet 378, 1388-1395 (2011).

8. P. Kahane, A. Depaulis, Deep brain stimulation in epilepsy: What is next? Curr. Opin. Neurol. 23, 177-182 (2010)

9. E. H. Kossoff, A. L. Hartman, Ketogenic diets: New advances for metabolism-based therapies. Curr. Opin. Neurol. 25, 173-178 (2012).

10. P. Boon, R. Raedt, V. de Herdt, T. Wyckhuys, K. Vonck, Electrical stimulation for the treatment of epilepsy. Neurotherapeutics 6, 218-227 (2009)

11. O. Devinsky, Sudden, unexpected death in epilepsy. N. Engl. J. Med. 365, 1801-1811 (2011)

12. C. Hoppe, C. E. Elger, Depression in epilepsy: A critical review from a clinical perspective. Nat. Rev. Neurol. 7, 462-472 (2011).

13. C. Richichi, E. J. Lin, D. Stefanin, D. Colella, T. Ravizza, G. Grignaschi, P. Veglianese, G. Sperk M. J. During, A. Vezzani, Anticonvulsant and antiepileptogenic effects mediated by adenoassociated virus vector neuropeptide $Y$ expression in the rat hippocampus. J. Neurosci. 24, 3051-3059 (2004)

14. D. P. Woldbye, M. Angehagen, C. R. Gøtzsche, H. Elbrønd-Bek, A. T. Sørensen, S. H. Christiansen M. V. Olesen, L. Nikitidou, T. V. Hansen, I. Kanter-Schlifke, M. Kokaia, Adeno-associated viral vector-induced overexpression of neuropeptide $\mathrm{Y} Y 2$ receptors in the hippocampus suppresses seizures. Brain 133, 2778-2788 (2010).

15. R. P. Haberman, R. J. Samulski, T. J. McCown, Attenuation of seizures and neuronal death by adeno-associated virus vector galanin expression and secretion. Nat. Med. 9, 1076-1080 (2003)

16. E. J. D. Lin, D. Young, K. Baer, H. Herzog, M. J. During, Differential actions of NPY on seizure modulation via Y1 and Y2 receptors: Evidence from receptor knockout mice. Epilepsia 47 773-780 (2006).

17. T. J. McCown, Adeno-associated virus-mediated expression and constitutive secretion of galanin suppresses limbic seizure activity in vivo. Mol. Ther. 14, 63-68 (2006).

18. I. Kanter-Schlifke, B. Georgievska, D. Kirik, M. Kokaia, Seizure suppression by GDNF gene therapy in animal models of epilepsy. Mol. Ther. 15, 1106-1113 (2007).

19. R. Bovolenta, S. Zucchini, B. Paradiso, D. Rodi, F. Merigo, G. Navarro Mora, F. Osculati, E. Berto P. Marconi, A. Marzola, P. F. Fabene, M. Simonato, Hippocampal FGF-2 and BDNF overexpression attenuates epileptogenesis-associated neuroinflammation and reduces spontaneous re current seizures. J. Neuroinflammation 7, 81 (2010).

20. F. Noè, A. H. Pool, J. Nissinen, M. Gobbi, R. Bland, M. Rizzi, C. Balducci, F. Ferraguti, G. Sperk, M. J. During, A. Pitkänen, A. Vezzani, Neuropeptide Y gene therapy decreases chronic spontaneous seizures in a rat model of temporal lobe epilepsy. Brain 131, 1506-1515 (2008).

21. E. D. Louis, P. D. Williamson, T. M. Darcey, Chronic focal epilepsy induced by microinjection of tetanus toxin into the cat motor cortex. Electroencephalogr. Clin. Neurophysiol. 75, 548-557 (1990).

22. K. E. Nilsen, M. C. Walker, H. R. Cock, Characterization of the tetanus toxin model of refractory focal neocortical epilepsy in the rat. Epilepsia 46, 179-187 (2005)

23. O. C. Cockerell, J. Rothwell, P. D. Thompson, C. D. Marsden, S. D. Shorvon, Clinical and physiological features of epilepsia partialis continua. Cases ascertained in the UK. Brain 119 (Pt. 2), 393-407 (1996)

24. P. Chang, K. S. Hashemi, M. C. Walker, A novel telemetry system for recording EEG in small animals. J. Neurosci. Methods 201, 106-115 (2011).

25. J. A. Blanco, M. Stead, A. Krieger, W. Stacey, D. Maus, E. Marsh, J. Viventi, K. H. Lee, R. Marsh B. Litt, G. A. Worrell, Data mining neocortical high-frequency oscillations in epilepsy and controls. Brain 134, 2948-2959 (2011).
26. S. J. Korn, J. L. Giacchino, N. L. Chamberlin, R. Dingledine, Epileptiform burst activity induced by potassium in the hippocampus and its regulation by GABA-mediated inhibition J. Neurophysiol. 57, 325-340 (1987).

27. G. Hagemann, M. Hoeller, C. Bruehl, M. Lutzenburg, O. W. Witte, Effects of tetanus toxin on functional inhibition after injection in separate cortical areas in rat. Brain Res. 818, 127-134 (1999).

28. M. Vreugdenhil, S. P. Hack, A. Draguhn, J. G. Jefferys, Tetanus toxin induces long-term changes in excitation and inhibition in the rat hippocampal CA1 area. Neuroscience 114 983-994 (2002).

29. M. Mainardi, M. Pietrasanta, E. Vannini, O. Rossetto, M. Caleo, Tetanus neurotoxin-induced epilepsy in mouse visual cortex. Epilepsia 53, e132-e136 (2012).

30. A. Groh, H. S. Meyer, E. F. Schmidt, N. Heintz, B. Sakmann, P. Krieger, Cell-type specific properties of pyramidal neurons in neocortex underlying a layout that is modifiable depending on the cortical area. Cereb. Cortex 20, 826-836 (2010).

31. H. Beck, Y. Yaari, Plasticity of intrinsic neuronal properties in CNS disorders. Nat. Rev. Neurosci. 9, 357-369 (2008).

32. J. Tønnesen, A. T. Sørensen, K. Deisseroth, C. Lundberg, M. Kokaia, Optogenetic control of epileptiform activity. Proc. Natl. Acad. Sci. U.S.A. 106, 12162-12167 (2009).

33. F. Zhang, L. P. Wang, M. Brauner, J. F. Liewald, K. Kay, N. Watzke, P. G. Wood, E. Bamberg G. Nagel, A. Gottschalk, K. Deisseroth, Multimodal fast optical interrogation of neural circuitry Nature 446, 633-639 (2007)

34. J. V. Raimondo, L. Kay, T. J. Ellender, C. J. Akerman, Optogenetic silencing strategies differ in their effects on inhibitory synaptic transmission. Nat. Neurosci. 15, 1102-1104 (2012).

35. A. E. Metz, N. Spruston, M. Martina, Dendritic D-type potassium currents inhibit the spike after depolarization in rat hippocampal CA1 pyramidal neurons. J. Physiol. 581, 175-187 (2007).

36. J. F. van Brederode, J. M. Rho, R. Cerne, B. L. Tempel, W. J. Spain, Evidence of altered inhibition in layer $\mathrm{V}$ pyramidal neurons from neocortex of Kcna1-null mice. Neuroscience 103, 921-929 (2001).

37. J. M. Bekkers, A. J. Delaney, Modulation of excitability by $\alpha$-dendrotoxin-sensitive potassium channels in neocortical pyramidal neurons. J. Neurosci. 21, 6553-6560 (2001).

38. J. H. Heeroma, C. Henneberger, S. Rajakulendran, M. G. Hanna, S. Schorge, D. M. Kullmann Episodic ataxia type 1 mutations differentially affect neuronal excitability and transmitter release. Dis. Model Mech. 2, 612-619 (2009).

39. A. S. Galanopoulou, P. S. Buckmaster, K. J. Staley, S. L. Moshé, E. Perucca, J. Engel Jr., W. Löscher, J. L. Noebels, A. Pitkänen, J. Stables, H. S. White, T. J. O'Brien, M. Simonato; American Epilepsy Society Basic Science Committee And The International League Against Epilepsy Working Group On Recommendations For Preclinical Epilepsy Drug Discovery, Identification of new epilepsy treatments: Issues in preclinical methodology. Epilepsia 53, 571-582 (2012).

40. A. Bragin, J. Engel Jr., R. J. Staba, High-frequency oscillations in epileptic brain. Curr. Opin Neurol. 23, 151-156 (2010)

41. G. A. Worrell, L. Parish, S. D. Cranstoun, R. Jonas, G. Baltuch, B. Litt, High-frequency oscillations and seizure generation in neocortical epilepsy. Brain 127, 1496-1506 (2004).

42. S. M. Rothman, The therapeutic potential of focal cooling for neocortical epilepsy Neurotherapeutics 6, 251-257 (2009)

43. J. D. Heiss, S. Walbridge, A. R. Asthagiri, R. R. Lonser, Image-guided convection-enhanced delivery of muscimol to the primate brain. J. Neurosurg. 112, 790-795 (2010).

44. E. M. Slimko, S. McKinney, D. J. Anderson, N. Davidson, H. A. Lester, Selective electrica silencing of mammalian neurons in vitro by the use of invertebrate ligand-gated chloride channels. J. Neurosci. 22, 7373-7379 (2002).

45. G. G. Turrigiano, The self-tuning neuron: Synaptic scaling of excitatory synapses. Cell 135 422-435 (2008).

46. G. Schiavo, F. Benfenati, B. Poulain, O. Rossetto, P. Polverino de Laureto, B. R. DasGupta C. Montecucco, Tetanus and botulinum-B neurotoxins block neurotransmitter release by proteolytic cleavage of synaptobrevin. Nature 359, 832-835 (1992).

47. H. Su, D. Sochivko, A. Becker, J. Chen, Y. Jiang, Y. Yaari, H. Beck, Upregulation of a T-type $\mathrm{Ca}^{2+}$ channel causes a long-lasting modification of neuronal firing mode after status epilepticus. J. Neurosci. 22, 3645-3655 (2002).

48. M. M. Shah, A. E. Anderson, V. Leung, X. Lin, D. Johnston, Seizure-induced plasticity of channels in entorhinal cortical layer III pyramidal neurons. Neuron 44, 495-508 (2004)

49. C. Bernard, A. Anderson, A. Becker, N. P. Poolos, H. Beck, D. Johnston, Acquired dendritic channelopathy in temporal lobe epilepsy. Science 305, 532-535 (2004).

50. P. A. Castro, E. C. Cooper, D. H. Lowenstein, S. C. Baraban, Hippocampal heterotopia lack functional Kv4.2 potassium channels in the methylazoxymethanol model of cortical malformations and epilepsy. J. Neurosci. 21, 6626-6634 (2001).

51. P. C. Bush, D. A. Prince, K. D. Miller, Increased pyramidal excitability and NMDA conductance can explain posttraumatic epileptogenesis without disinhibition: A model. J. Neurophysiol. 82 1748-1758 (1999).

52. L. Topolnik, M. Steriade, I. Timofeev, Hyperexcitability of intact neurons underlies acute development of trauma-related electrographic seizures in cats in vivo. Eur. J. Neurosci. 18, 486-496 (2003). 
53. M. M. Jagodic, S. Pathirathna, M. T. Nelson, S. Mancuso, P. M. Joksovic, E. R. Rosenberg D. A. Bayliss, V. Jevtovic-Todorovic, S. M. Todorovic, Cell-specific alterations of T-type calcium current in painful diabetic neuropathy enhance excitability of sensory neurons. J. Neurosci. 27, 3305-3316 (2007)

54. H. J. Hu, Y. Carrasquillo, F. Karim, W. E. Jung, J. M. Nerbonne, T. L. Schwarz, R. W. Gereau IV The kv4.2 potassium channel subunit is required for pain plasticity. Neuron 50, 89-100 (2006).

55. R. Wykes, A. Kalmbach, M. Eliava, J. Waters, Changes in the physiology of CA1 hippocampal pyramidal neurons in preplaque CRND8 mice. Neurobiol. Aging 33, 1609-1623 (2012).

56. D. C. Rogers, C. A. Campbell, J. L. Stretton, K. B. Mackay, Correlation between motor impairment and infarct volume after permanent and transient middle cerebral artery occlusion in the rat. Stroke 28, 2060-2065 (1997).

57. T. Hedrick, J. Waters, Spiking patterns of neocortical L5 pyramidal neurons in vitro change with temperature. Front. Cell. Neurosci. 5, 1 (2011).

Acknowledgments: We thank G. Schiavo (Cancer Research UK) for the gift of tetanus toxin F. Gaba (UCL) for blinded seizure counting, and I. Oren (UCL) for help with data analysis code. We are also grateful to M. E. Collins and D. Escors for supervising lentivirus production and to J. Parnavelas, A. Cariboni, F. Chiara, and S. Davies for advice on immunohistochemistry and access to equipment. We thank G. Ritter and C. Ramakrishnan for help with the laser setup and NpHR plasmid, and D. Kaetzel for comments on the manuscript. Funding: This work was supported by the Medical Research Council, the Wellcome Trust, the European Research Council, the Royal Society, the Guarantors of Brain, the Brain Research Trust, and Epilepsy Research UK. Author contributions: R.C.W., J.H.H., and L.M. performed in vivo experiments and histology and analyzed EEG. The NpHR lentivirus was made by L.M., D.C.M., and K.D. In vitro electrophysiology was carried out by R.C.W. The epileptiform event detection was optimized by J.H.H., K.Z., and K.S.H. M.C.W., S.S., and D.M.K. supervised the study and drafted the manuscript. Competing interests: K.S.H. is president of Open Source Instruments Inc., the company that assembles the subcutaneous transmitters with which the EEG recordings were made in this study. The other authors declare no competing interests. Data and materials availability: All source code and circuit diagrams are available from http://www.opensourceinstruments.com EEG data are available on request.

Submitted 18 April 2012

Accepted 5 October 2012

Published 21 November 2012

10.1126/scitranslmed.3004190

Citation: R. C. Wykes, J. H. Heeroma, L. Mantoan, K. Zheng, D. C. MacDonald, K. Deisseroth K. S. Hashemi, M. C. Walker, S. Schorge, D. M. Kullmann, Optogenetic and potassium channel gene therapy in a rodent model of focal neocortical epilepsy. Sci. Transl. Med. 4, $161 \mathrm{ra} 152$ (2012). 


\section{Abstracts}

One-sentence summary: Light-activated gene therapy acutely suppresses seizures, and gene therapy with a potassium channel prevents epileptogenesis and treats established epilepsy in a rodent model.

\section{Editor's Summary: \\ Casting Light on the Shadow of Epilepsy}

Epilepsy affects $1 \%$ of the population and is often resistant to medication. Surgery to remove the region of the brain that generates seizures is only feasible in a minority of cases because of risks to movement, language, vision, and other essential functions. There is an urgent need for alternative treatments. In a new study, Wykes et al. used a virus to express a therapeutic gene in a small number of brain neurons in the seizure-generating zone in a rat model of drug-resistant epilepsy. They also developed new wireless technology to monitor and detect seizures using a miniaturized implanted transmitter and advanced algorithms. They took two approaches to reduce brain circuit excitability and hence epileptic seizures in the rat model. First, to suppress neuronal firing acutely, they expressed the light-sensitive chloride transporter halorhodopsin in the seizure-generating zone. When laser light was delivered via an optic fiber to this region and the halorhodopsin was activated, they observed a decrease in electrical seizure activity. The success of this "optogenetic" approach implies that a device could be developed to detect and stop seizures "on demand" akin to an implantable defibrillator for heart rhythm disturbances. For longer-term suppression of epilepsy, the investigators overexpressed a brain potassium ion channel that normally regulates both neuronal excitability and neurotransmitter release. This gene therapy treatment fully prevented epilepsy from developing in the rat model. When it was applied during established epilepsy, potassium channel gene therapy progressively diminished the frequency of seizures until they stopped after a few weeks. Neither of the gene therapy approaches tested interfered with normal behavior, most likely because only a small number of neurons were targeted. Although still in the earliest stages of study, this gene therapy approach may hold promise for treating drug-resistant epilepsy. 